\title{
EXPLORING THE ROLE OF TRANSACTIVE MEMORY SYSTEMS IN TEAM DECISION-MAKING DURING IDEATION PHASE
}

\author{
Dastmalchi, Mohammad Reza; \\ Balakrishnan, Bimal; \\ Oprean, Danielle
}

University of Missouri

\begin{abstract}
Team collaboration is a critical necessity of the modern-day engineering design profession. This is no surprise given that teams typically possess more task-relevant skills and knowledge than individuals (Levine \& Choi, 2004). Advancements in digital media provide new opportunities for collaboration across the design lifecycle. However, early stages of the design process still pose challenges to digitally mediated design collaboration due to greater representational abstraction and the presence of multiple modalities for design ideation. Usually, design teams spend a substantial amount of time generating a broad set of ideas that can lead them to a wide range of design solutions during the ideation phase. However, sooner or later, teams should narrow down their vision for a final solution. What factors influence team members to eliminate or select an idea? Our study is an attempt to demonstrate some examples of this challenge. By drawing on research in team cognition, particularly the concept of transactive memory system (TMS) we studied a design teams' communication and media use during the ideation phase. The goal was to see if media type and communication modes can predict a team's decisions on selecting and eliminating ideas.
\end{abstract}

Keywords: Collaborative design, Design cognition, Communication, Team Cognition

\section{Contact:}

Dastmalchi, Mohammad Reza

University of Missouri

Architectural Studies

United States of America

dastmalchim@mail.missouri.edu 


\section{INTRODUCTION}

In the past few decades, design practice has become more collaborative due to several reasons. These include an increase in the number of specializations within the design industry and globalization that resulted in a wider geographic distribution of design practice. Advancement of technology, such as higher bandwidth for data transmission (i.e., optic fiber and 5G), and the development of new digital tools that support teamwork further accelerated this trend. Considering that teams possess more taskrelevant skills and knowledge than individuals (Levine and Choi, 2004), it is no surprise that design teams have become more common among organizations. With the increase in globalization and competition, there has been more demand on design teams to create more novel and innovative designs. To do so, usually, design teams spend a substantial amount of time generating numerous ideas that can lead them to a wide range of design solutions. However, sooner or later, teams should narrow down their vision for a final solution. What factors influence team members to eliminate or select ideas? This is a broad question, and many possible answers could exist. Since this inquiry has received minimal attention within the engineering design community of scholars, our main intention was to create an opportunity through which, engineering design and design thinking researchers discuss and explore the subject. In order to demonstrate our approach, we used a case study and further analysed our preliminary findings. In this article, we are reporting our approach and findings on possible role of different communication modes and media on this process. Specifically, we were interested in finding out more about media that could be used as a shared (or team-operated) device, such as a shared display, compared to individually operated systems, such as individual desktops. We studied a design team's communication and interactions as they were collaborating on a project. Our main hypothesis was based on the theories of team cognition, specifically Transactive Memory Systems (TMS). TMS is the system used by a team for encoding, storing, and retrieving knowledge across different domains (Ren and Argote, 2011). It is widely believed that TMS can enhance team performance by improving team communication and coordination (Badke-Schaub et al., 2007). However, our knowledge on the impact of TMS on decision making is limited. By using protocol analysis (Ellis, 2006), we assessed the relationship between TMS and decision-making during ideation phase. By decision-making, we are referring to team members' decisions on selecting and eliminating ideas, which usually occurs at the end or after the ideation phase.

\section{TRANSACTIVE MEMORY SYSTEMS AND CODING SCHEMES}

Wegner (1987) first coined the term transactive memory and defined it as a "shared system for encoding, storing, and retrieving information" (Wegner et al., 1991, p. 923). Since then, the conceptualization of the transactive memory system has developed along two complementary approaches. These approaches reflect the cognitive and behavioural dimensions of the construct (Ellis, 2006). The cognitive side of TMS operationalizes it as team members' agreement about specialization, the credibility of team members, and coordination (Lewis, 2003). The behavioural side of TMS focuses on directory updating, information allocation, and retrieval coordination (Ellis, 2006). The general thesis of TMS is that it can help teams improve problem-solving by giving access to more efficient and larger pools of stored information and a much faster recall of relevant information to address an issue (Ren et al., 2006). Previous studies have operationalized TMS as an external memory accessible to everyone; in a specific case, it was the internet (Sparrow and her associates, 2011).

In design, we communicate our ideas through design representations, such as sketches, prototypes, and many others. There are different ways that design representation impacts our communication and process. Design representations help us by storing and holding information as we think and evaluate various aspects of the design. This means design representations help us overcome the cognitive limitations of our working memory; hence, they act as external memories. Design knowledge is multimodal and often multiple representations are required to understand a design. We conceptualized TMS as the system that encodes, stores, retrieves, and transfers new information. In this case, by information or data, we refer to the representation of newly proposed ideas that team members generate. Therefore, in this research study, we operationalize TMS as 1) the system used for storing and accessing the information, 2) presentation platforms used for communicating information; and 3) the modes of design representations 4) and media used for externalizing and encoding the design representations. 
Two different systems were used for storing and retrieving the information, which was cloud storage and USB flash drive. The team had three platforms through which they could present their work, namely projector, whiteboard, and computer desktop. Throughout the process, besides the verbal mode, sketch, textual, and digital images were used as modes of communication. Three different media tools were provided for the team to encode their ideas with, including personal journals, software (such as Microsoft Word and PowerPoint), and E-Beam Capture, which was used to capture the content of the whiteboard and transform it into a PDF file. Figure 1 shows the operationalization that was used for coding TMS. The system that was used in the collaborative environment where either a commonly operated system (marked as "(C)", such as shared display for projector) or individually operated system (marked as "(I)", such personal journals/notebook).

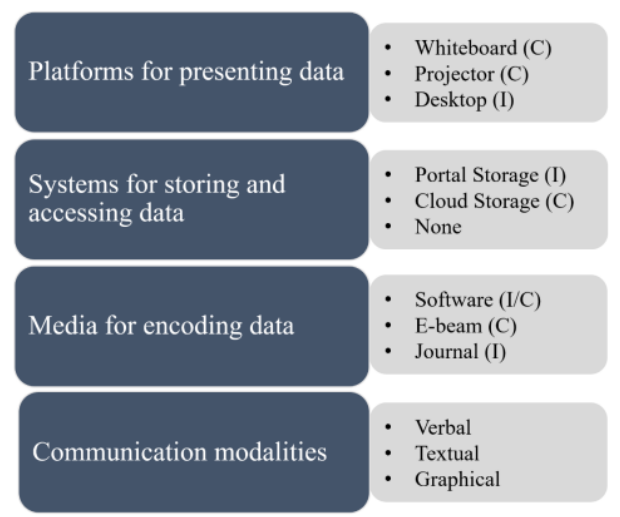

Figure 1. Operationalization of TMS, items with (C) functioned at a team level and items with (I)

In addition to TMS, we also coded 'new ideas', by operationalizing them as 'direct and indirect discussion about a new idea, that was not discussed before'; 'introducing a new idea' (Gabriel and Maher 2002). Furthermore, any idea that was part of the team's final presentation was considered as 'selected ideas' and the ones that were omitted were considered as 'eliminated'.

\section{RESEARCH QUESTIONS AND METHOD}

Considering the importance of TMS in design communication, coordination, and creativity (Kleinsmann and Valkenburg, 2008), we wanted to know if TMS has a relationship with decisionmaking towards the selection and elimination of ideas during the ideation phase. In order to measure TMS, we used protocol analysis (Ericsson and Simon, 1993). Two researchers coded the data by using 'new ideas' as units of analysis. TMS was seen as a function of media affordances (Balakrishnan and Oprean, 2015). Different tools and media were available to the team to facilitate collaboration. We examined how different media affordances contributed to encoding, storing, and retrieving information; in other words, how did different tools and media as external memories contribute to the process of idea selection and elimination. By tracking each 'idea' throughout the design process, we coded how each idea was introduced; the modalities through which the idea was discussed, the presentation platforms used when the idea was discussed, and what system was used to store and access the idea. Therefore, we proposed the following question next:

RQ: Which dimensions of TMS correlates with the teams' decision-making in idea selection?

Based on this question we proposed four hypotheses. According to our first hypothesis, presentation platforms that functioned at a team level are predictors of idea selection. This is based on the fact that shared displays improve team coordination and therefore they are more effective in team decisionmaking (McNeese, et al., 2006; Bolstad and Endsley, 1999). The second hypothesis stated information that was stored/accessible for the entire team (cloud storage) are predictor of idea selection. The third and fourth hypothesis was based on media used for encoding data, and modes of communication. However, previous research on this area have reported various contradictory results. A series of experiments were conducted in the 1990s assessing the advantages of multimodal and multimedia instructions. Multimodality refers to the idea of someone engage more than one sense modality to communicate, such as text, audio, video; while multimedia refers to the concept of integrating multiple media for presentation, such as animation and narration (Mayer and F Sims, 1994). For example, in an empirical study, four groups of participants learned about how a bicycle pump works using animation 
along with simultaneous audio and text (group 1), only audio and text (group 2), only animation and no audio (group 3), and no formal training as the control group (group 4). According to Mayer and Anderson (1991), the study results suggest that group 1 outperformed the rest of the groups. Other studies indicate that audio/video instructions may only be effective when the two modes are presented simultaneously instead of sequentially (Mayer and Anderson, 1992; Mayer and Sims, 1994). In a different study, researchers (Davis, et al., 2006) assessed the impacts of technology on jury decisionmaking. The participant used different modalities of the suspect statements, such as text, audio, or video. Their result suggested that audio and video users were more accurate in their decision-making (detecting lies) based on the present evidence. Based on these findings, we hypothesized that using multiple tools and modalities to discuss about an idea would increase the likely hood of that idea being selected.

\section{COLLABORATIVE ENVIRONMENT AND TOOLS}

The collaborative environment used for this study included a system (see Balakrishnan and Oprean, 2015) that draws on the role of design representation in the creative process, See. The implication of such collaborative environment that supports digitally mediated communication impacts the team's coordination, through better information flow. The idea behind this kind of collaborative environment is that multimodal information can help users overcome their working memory limitations, which can result in better learning and decision-making. The limitations of working memory have been the subject of studies for many decades. Working memory can only hold limited amounts of information for a short duration. Having too many pieces of information may result in decreased effectiveness for processing in working memory (Kalyuga et al., 1999). The team's primary collaborative workspace included 12 feet by 8 feet, digitally augmented whiteboard capable of sharing digital content powered by a graphics workstation. It also could capture various interactions and annotations digitally. Figure 2 demonstrates the setup that was used by the design team. The captured content was stored and accessible through cloud storage. Moreover, a projector was available to augment digital content through a desktop Additionally, each team member had access to a computer.

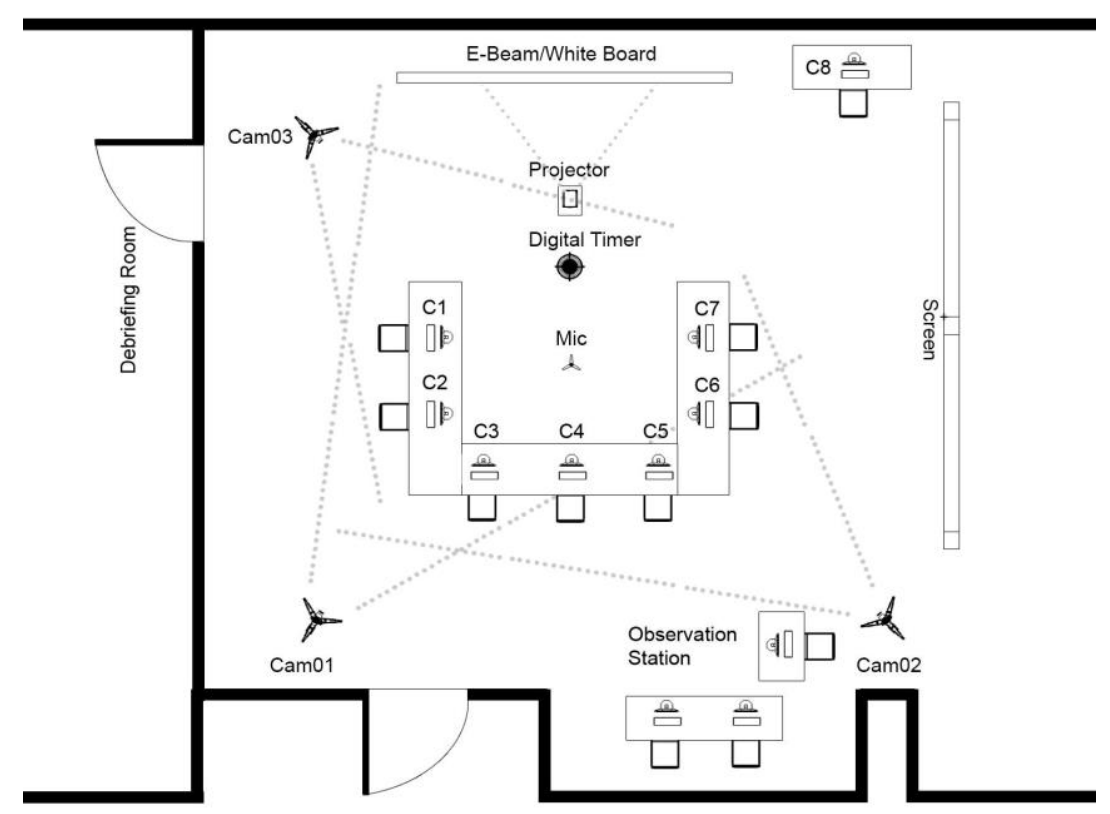

Figure 2. Collaborative environment setup

\section{PROJECT BACKGROUND AND DATA}

Our project documented a design process involving a multidisciplinary design team with seven members (for more information see D'souza and Dastmalchi, 2016). Team members had different disciplinary affiliations, expertise, and knowledge levels collaborated on a design project. The task was to design a way, whether a product or service, to celebrate a birthday in the 21 st century. The design brief was proposed by a Mid-Western greeting card company interested in finding unique ways 
of celebration that goes beyond the idea of birthday cards. The entire creative project spanned five days; Day one included the project briefing. On day two, the team conducted idea generation sessions, which lasted for 90 minutes. On day three, the team went through the process of eliminating some ideas, and organizing and categorizing ideas selected for development, which lasted for four hours. During day four, the team executed their ideas, and finally, on day five, they presented their ideas at the company headquarter. Table below shows an overview of the events and the used data for this study has been highlighted. Every step of this process was video recorded. The videos consist of 15 sessions in total, each lasts 40 to 50 minutes long. For this study, we used 90 minutes of video data of the team's collaboration on day 2 , and the first 40 minutes of day 3 - as it was the time devoted to idea selection, as shown in table 1 . And finally, the transcription of the videos, screen captures, and team members' individual journals were also used.

Table 1. Summary of data

\begin{tabular}{|l|l|l|l|l|l|}
\hline & Day 1 & Day 2 & Day 3 & Day 4 & Day 5 \\
\hline $\begin{array}{l}\text { Event and } \\
\text { Location }\end{array}$ & $\begin{array}{l}\text { Debriefing } \\
\text { @ HQ }\end{array}$ & Ideation @ Lab & $\begin{array}{l}\text { Idea selection, } \\
\text { organizing \& } \\
\text { planning @ Lab }\end{array}$ & $\begin{array}{l}\text { Executing Prototypes } \\
\text { \& Presentation prep } \\
\text { @ Lab }\end{array}$ & $\begin{array}{l}\text { Presentation @ } \\
\text { HQ }\end{array}$ \\
\hline $\begin{array}{l}\text { Number of } \\
\text { Sessions }\end{array}$ & 4 & 2 & 6 & 7 & 1 \\
\hline $\begin{array}{l}\text { Total Footage } \\
\text { Time }\end{array}$ & 3 Hours & $\begin{array}{l}\text { 1 Hour and 30 } \\
\text { Minutes }\end{array}$ & 4 Hours & $\begin{array}{l}\text { 4 Hours and 40 } \\
\text { Minutes }\end{array}$ & 3 Hours \\
\hline Data Use & - & $\begin{array}{l}\text { Full time video } \\
\text { and screen } \\
\text { capture }\end{array}$ & $\begin{array}{l}\text { [First] 40 minutes } \\
\text { of video and screen } \\
\text { capture }\end{array}$ & - & - \\
\hline
\end{tabular}

\section{RESULTS AND DISCUSSION}

Two individual coders used INTERACT software for coding purposes. The interrater reliability shows $\kappa=0,93$ on TMS and $\kappa=0,88$ on 'new ideas'. Our reported results are the outcome of a single case study, and therefore we do not intend to generalize the findings. Figure 3 shows how total of 48 ideas was introduced and discussed. Furthermore, selected ideas are highlighted and eliminated ideas are shaded.

To test our hypothesis about TMS, we performed a Chi-Square test of independence to check for differences among various presentation platforms, communication modality, encoding medium, and data storage systems in relation to idea selection. Presentation platforms varied from common/public (or team operated) to individual. Common image platforms were visible to everyone from anywhere within the collaborative environment, such as a projector or the whiteboard. Individual platforms, on the other hand, required members to use their individual devices, such as desktops, to see websites or images for example. We hypothesized that ideas that were presented from a shared platform should be more likely to be selected. A significant difference was found between ideas that had no specific presentation platform and were eliminated compared to other ideas. Additionally, ideas that were presented through the whiteboard platform were a predictor of idea selection, $X^{2}(8, N=48)=73.53$, $\mathrm{p}<0.001$. In terms of information transmission and data storage, our analysis indicated that cloud storage was utilized more than portable storage (USB flash memory) during the ideation phase. Therefore, using cloud storage as a means to store and access information increased the likelihood of ideas being selected, $\mathrm{X}^{2}(4, \mathrm{~N}=48)=70.17, \mathrm{p}<0.001$. In terms of media used for encoding information, journals and software were used at an individual level. In other words, a member would encode the data or 'take notes' from the ideas that were under discussion. This encoding occurred through the use of personal media (personal computer using a Word document or personal journal). The encoded ideas (notes that were taken) were the interpretation of the member who was encoding the information (i.e., taking the note) and not necessarily the person who was discussing or proposing the idea.

Additionally, the data encoded via software and journals were only available to the owner of the device, unless he/she somehow shared their notes with everyone. E-beam, on the other hand, was the medium that operated at a team level, meaning everything that was encoded through E-beam was a product of team discussion and the content recorded by this device was a result of team activity. Moreover, the information encoded by the E-beam was immediately available to everyone through the cloud storage. In other words, everyone had easy access to the content encoded via E-beam from their personal computers. Our results show a significant difference between selected ideas that were encoded via E-beam, $\mathrm{X}^{2}(4, \mathrm{~N}=48)=75.49, \mathrm{p}<0.001$. We believe using such a shared system as $\mathrm{E}$ beam will provide a great opportunity to externalize ideas and store them for later. In fact, our further 
investigation concluded that the content that was captured by the E-beam was frequently accessed and used by the team members. We found that E-beam content was viewed during the idea selection session for a total of 23 minutes. Additionally, among different modes of communication, sketching was found to be a predictor for idea selection $X^{2}(1, N=37)=69.78, p<0.001$. No association was found between the textual or digital image and idea selection. Furthermore, the ideas that were encoded through multiple modes were also predictors of idea selection, $\mathrm{X}^{2}(1, \mathrm{~N}=37)=70.28$, $\mathrm{p}<0.001$. According to the previous studies however, receiving information from multiple human channels can work as a 'double-edged sword' - it can hinder the user's memory in some content, or it can positively influence it (Sundar, 2000).

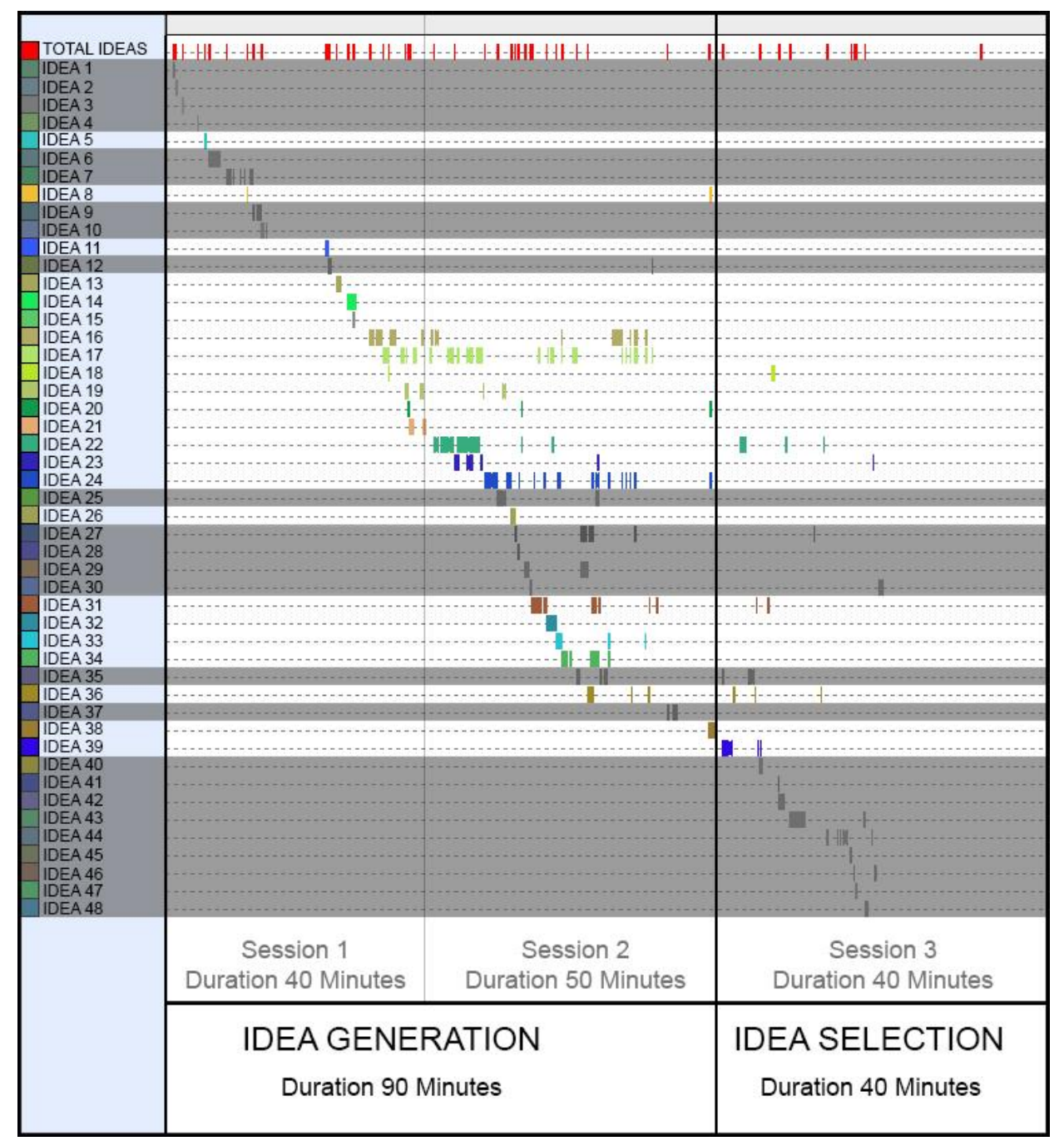

Figure 3. Distribution of generated, selected, and eliminated ideas throughout different sessions

Our next hypothesis was that the number of ideas proposed in the last session and was selected as part of the final product is significantly different from the rest of the selected ideas. The limitations of working memory have been the subject of studies for many decades. Based on the working memory model, any increase in the cognitive processes of new information that is not directly linked to the acquisition of new schemas would result in a burden of working memory capacity and reduction of resources one uses for learning. However, our results indicate that in fact, most of the ideas that were selected were produced during the second session of idea generation. More than half of the selected ideas $(56.5 \%)$ were produced during the second session, while the first session held $31 \%$ and the third session held $12.5 \%$ of the selected ideas. Table 2 shows the total number of ideas that was generated in each session, and how many of the selected ideas were originally generated in the respected session. Therefore, as the team was going through the process of selecting ideas for their final design solution, some ideas were produced and even one selected, but anything proposed after that was never considered. 
Table 2. Number of generated, selected, and eliminated ideas throughout different sessions (\# in frequency of occurrence)

\begin{tabular}{|l|c|c|c|}
\hline Event (Duration) & \multicolumn{2}{|c|}{ IDEA GENERATION } & $\begin{array}{c}\text { IDEA } \\
\text { SELECTION }\end{array}$ \\
\hline $\begin{array}{l}\text { Session \# } \\
\text { (Duration) }\end{array}$ & Session 1 & Session 2 & Session 3 \\
\hline Ideas Generated \# & 18 & 21 & 9 \\
\hline Selected Ideas \# & 9 & 13 & 1 \\
\hline Eliminated idea \# & 9 & 8 & 8 \\
\hline
\end{tabular}

\section{SUMMARY AND FUTURE RESEARCH}

Our main goal was to highlight the subject of decision-making during ideation phase by demonstrating some examples. Since we only used one case study we cannot generalize or make further interpretations of the results. By drawing on the theories of team cognition, we hypothesized that tools and modes of communication can predict decision on selecting ideas. By operationalizing TMS as different forms of media, we found a that various modes of communication can predict decisionmaking during ideation phase. Specifically, we found multi modal and sketching a predictor of idea selection. Furthermore, media such as E-beam/whiteboard, projector, and cloud storage which operated at a team level, contrary to those that operate individually, were also predictors of idea selection. This is important because affordances of such tools can impact team coordination and performance (McNeese, et al., 2006; Bolstad and Endsley, 1999). It would be ideal if the future studies can expand upon this subject, especially by conducting experiments where media can be systematically manipulated. Moreover, by using more case studies in the future, future studies can compare team performance and other constructs of team cognition such as situation awareness and team mental model. Mohammed and her colleagues (2010), pointed out the importance and lack of studies where multiple constructs of team cognition is measured (such as Ellis, 2006).

\section{REFERENCES}

Balakrishnan, B. and Oprean, D., 2015. Communication, Coordination and Collaboration: Media Affordances and Team Performance in a Collaborative Design Environment. In eCAADe 2015- real time-extending the reach of computation: proceedings of the 33rd International Conference on Education and Research in Computer Aided Architectural Design in Europe (Vol. 2, pp. 225-231). eCAADe.

Bolstad, C.A. and Endsley, M.R., 1999, September. Shared mental models and shared displays: An empirical evaluation of team performance. In proceedings of the human factors and ergonomics society annual meeting (Vol. 43, No. 3, pp. 213-217). Sage CA: Los Angeles, CA: SAGE Publications.

Brandon, D.P. and Hollingshead, A.B., 2004. Transactive memory systems in organizations: Matching tasks, expertise, and people. Organization science, 15(6), pp.633-644.

Choi, S.Y., Lee, H. and Yoo, Y., 2010. The impact of information technology and transactive memory systems on knowledge sharing, application, and team performance: A field study. MIS quarterly, pp.855-870.

Converse, S., Cannon-Bowers, J.A. and Salas, E., 1993. Shared mental models in expert team decision making. Individual and group decision making: Current issues, 221, pp.221-46.

Cooke, N.J., Salas, E., Cannon-Bowers, J.A. and Stout, R.J., 2000. Measuring team knowledge. Human factors, 42(1), pp.151-173.

Davis, M., Markus, K.A. and Walters, S.B., 2006. Judging the credibility of criminal suspect statements: does mode of presentation matter?. Journal of Nonverbal Behavior, 30(4), pp.181-198.

De Dreu, C.K. and Weingart, L.R., 2003. Task versus relationship conflict, team performance, and team member satisfaction: a meta-analysis. Journal of applied Psychology, 88(4), p.741.

D'souza, N. and Dastmalchi, M.R., 2016. Creativity on the move: Exploring little-c (p) and big-C (p) creative events within a multidisciplinary design team process. Design Studies, 46, pp.6-37.

Ellis, A.P., 2006. System breakdown: The role of mental models and transactive memory in the relationship between acute stress and team performance. Academy of Management Journal, 49(3), pp.576-589.

Gabriel, G.C. and Maher, M.L., 2002. Coding and modelling communication in architectural collaborative design. Automation in construction, 11(2), pp.199-211.

Hollingshead, A.B., 1998. Communication, learning, and retrieval in transactive memory systems. Journal of experimental social psychology, 34(5), pp.423-442.

Jehn, K.A., 1995. A multimethod examination of the benefits and detriments of intragroup conflict.

Administrative science quarterly, pp.256-282. 
Kalyuga, S., Chandler, P. and Sweller, J., 1999. Managing split-attention and redundancy in multimedia instruction. Applied Cognitive Psychology: The Official Journal of the Society for Applied Research in Memory and Cognition, 13(4), pp.351-371.

Kleinsmann, M. and Valkenburg, R., 2008. Barriers and enablers for creating shared understanding in co-design projects. Design studies, 29(4), pp.369-386.

Klimoski, R. and Mohammed, S., 1994. Team mental model: Construct or metaphor?. Journal of management, 20(2), pp.403-437.

Langan-Fox, J., Anglim, J. and Wilson, J.R., 2004. Mental models, team mental models, and performance: Process, development, and future directions. Human Factors and Ergonomics in Manufacturing \& Service Industries, 14(4), pp.331-352.

Lewis, K., 2003. Measuring transactive memory systems in the field: scale development and validation. Journal of applied psychology, 88(4), p.587.

Mayer, R.E. and Anderson, R.B., 1991. Animations need narrations: An experimental test of a dual-coding hypothesis. Journal of educational psychology, 83(4), p.484.

Mayer, R.E. and Anderson, R.B., 1992. The instructive animation: Helping students build connections between words and pictures in multimedia learning. Journal of educational Psychology, 84(4), p.444.

Mayer, R.E. and Sims, V.K., 1994. For whom is a picture worth a thousand words? Extensions of a dual-coding theory of multimedia learning. Journal of educational psychology, 86(3), p.389.

McNeese, M.D., Pfaff, M.S., Connors, E.S., Obieta, J.F., Terrell, I.S. and Friedenberg, M.A., 2006, October. Multiple vantage points of the common operational picture: Supporting international teamwork. In Proceedings of the human factors and ergonomics society annual meeting (Vol. 50, No. 3, pp. 467-471). Sage CA: Los Angeles, CA: SAGE Publications.

Mohammed, S., Ferzandi, L. and Hamilton, K., 2010. Metaphor no more: A 15-year review of the team mental model construct. Journal of management, 36(4), pp.876-910.

Oshri, I., van Fenema, P.C. and Kotlarsky, J., 2008. Knowledge transfer in globally distributed teams: the role of transactive memory. In Knowledge Processes in Globally Distributed Contexts (pp. 24-52). Palgrave Macmillan, London.

Ren, Y. and Argote, L., 2011. Transactive memory systems 1985-2010: An integrative framework of key dimensions, antecedents, and consequences. Academy of Management Annals, 5(1), pp.189-229.

Ren, Y., Carley, K.M. and Argote, L., 2006. The contingent effects of transactive memory: When is it more beneficial to know what others know?. Management Science, 52(5), pp.671-682.

Sparrow, B., Liu, J. and Wegner, D.M., 2011. Google effects on memory: Cognitive consequences of having information at our fingertips. science, 333(6043), pp.776-778.

Sundar, S.S., 2000. Multimedia effects on processing and perception of online news: A study of picture, audio, and video downloads. Journalism \& Mass Communication Quarterly, 77(3), pp.480-499.

Wegner, D.M., 1987. Transactive memory: A contemporary analysis of the group mind. In Theories of group behavior (pp. 185-208). Springer, New York, NY.

Wegner, D.M., Erber, R. and Raymond, P., 1991. Transactive memory in close relationships. Journal of personality and social psychology, 61(6), p.923. 